\title{
POST-VACCINIAL ENCEPHALITIS:
}

\section{RECOVERY FOLLOWING TREATMENT WITH INTRAVENOUS PENTOTHAL SODIUM AND CONVALESCENT SERUM}

BY

\author{
C. L. DAVIDSON, M.D., M.R.C.P., D.C.H., AND \\ JEAN TERRY THOMAS, M.B., Ch.B.
}

(From the Children's Department, Leeds General Infirmary).

Encephalitis has now been recognized for many years as a rare but serious complication of vaccination and some hundreds of cases have been reported. Its incidence is mainly in older children and young adults undergoing primary vaccination and it is extremely rare in the first year of life. Thus, in a serious outbreak in Holland in 1929 there were eighty-three cases resulting from about one and a quarter million vaccinations, none of which affected infants under the age of twelve months (Brain, 1940). In this country only eight cases in infants were reported to the Ministry of Health in the eight years from 1932 to 1939 , and of these five were fatal. The rarity and seriousness of the condition are thus evident.

The following case, in which recovery occurred in an infant aged four months, is recorded for two reasons-firstly because of its rarity, and secondly to draw attention to the efficacy of pentothal sodium and convalescent serum in treatment. Pentothal sodium is a valuable addition to the armamentarium of the paediatrician in the control of convulsions in infants and small children and has, in our experience, brought an end to intractable convulsions in three infants in whom all other measures had proved ineffective.

\section{Case report}

A first-born male infant of four months was admitted to the Leeds General Infirmary under the care of Prof. C. W. Vining on May 30, 1941. Its previous health and development had been satisfactory. On May 16 vaccination had been performed by a single scratch of a quarter of an inch on the left upper arm without any unusual local inflammatory reaction occurring. The infant's general condition had also remained satisfactory until May 27, when it became restless and was noticed to be making spasmodic movements of the head and eyes to the left. During the next two days its general attitude became one of gradually increasing muscular rigidity, and, though drowsy, it seemed to recognize its parents and would follow them with its eyes. Feeds had been taken fairly well, with vomiting on one occasion only, up to the time of admission on May 30. There had been some recent loss of weight. 
On admission the infant appeared ill. Its eyes were widely staring, and, although restless, it was clearly out of touch with its surroundings, and did not recognize anyone or respond to visual stimuli. Muscular tone was generally increased, and there were, in addition, repeated jerking movements of the head and eyes to the left, and occasional flexor spasms of the thighs on the abdomen. Perhaps the most striking feature was a rapid respiratory rhythm varying between fifty and sixty movements per minute and associated with inspiratory stridor. There were no signs to suggest meningitis, the fontanelle was not bulging, and examination of the discs and fundi showed nothing abnormal. The temperature and pulse rate on admission were $98.4^{\circ} \mathrm{F}$. and 132 per minute respectively. In spite of its condition the infant was able to swallow and, in addition to feeds of a dried milk mixture, was given one grain of chloral every four hours.

On the following morning the child's condition had deteriorated considerably. Restlessness was more severe and associated with short attacks of generalized convulsions in spite of increasing doses of bromide and chloral; rigidity of the limbs remained. The increase in respiratory rate had become more marked and of unusual character in that no pause followed either inspiration or expiration, the appearances suggesting a central stimulation of the respiratory centre. Lumbar puncture was performed and clear fluid obtained under normal pressure ; cytological and chemical examination of the fluid showed no abnormalities.

During the day chloroform inhalations were repeatedly given in an attempt to control restlessness and convulsions, but with only slight general effect and with none upon the respiratory rhythm. At 5.0 p.m. morphine $1 / 48$ grain was given, without response, and shortly afterwards there was regurgitation of altered blood from the stomach, which recurred at frequent intervals in the next three hours. At this stage the infant appeared moribund, sunken-eyed, pale and cyanosed, but still restless with a respiration rate of 90 to 100 per minute. The temperature was $103^{\circ} \mathrm{F}$. and the pulse rate 180 .

In the meantime $10 \mathrm{oz}$. of whole blood had been obtained from each of two probationer nurses who had been successfully vaccinated three to four weeks previously. The serum was obtained by centrifuge, and, in view of the seriousness of the condition, it was decided to administer it intravenously.

At 7.0 p.m. an intravenous infusion of glucose saline was begun, using a vein at the bend of the right elbow. General restlessness and tachypnoea were still very marked, and for this reason $0.025 \mathrm{gm}$. sodium pentothal was given with the first 2 to $3 \mathrm{oz}$. of fluid as it was feared that the continued muscular activity might, in itself, cause a fatal issue. The effect was immediate and dramatic. Within a minute or two general muscular relaxation occurred, and respiration, though still rapid, became quiet and peaceful; it was as though natural sleep had suddenly supervened on a severe delirium. During the next two hours $5 \mathrm{oz}$. of serum were given, followed by glucose saline to a total of $15 \mathrm{oz}$. Throughout the night the child remained peaceful, and its condition was greatly improved on the following day; it was able to take feeds by mouth though still stuporose. No further convulsions occurred, but the respiration rate remained high.

On June 2, the temperature fell to normal and the general improvement was maintained. No particular abnormalities were noted on examination though the child remained uninterested in its surroundings. It was discharged on June 4, six days after admission, apparently well.

The child was seen again on November 18,1941 , at the age of ten months. It then weighed $16 \frac{1}{2} \mathrm{lb}$. and was taking a mixed diet. The parents reported steady progress since discharge from hospital and they considered the mental 
condition to be satisfactory. The child was not then able to sit up with certainty, but was attempting to find its feet and was generally very active.

Physical examination revealed no particular abnormalities. All the limbs were moved actively, the fontanelle was open but not bulging, and in general, apart from some slight reduction in general nutrition, the child appeared normal and showed no sequelae of its illness.

\section{Comment}

In the consideration of this case two matters seem to call for discussion; first, the accuracy of the diagnosis, and secondly, the treatment employed. The circumstances of the case were such that it is felt that the diagnosis of postvaccinial encephalitis was fully justified. Recurrent convulsions, without apparent cause, even when repeated and sufficiently severe to cause death, are not rare in infancy, but in view of the gradual onset eleven days after vaccination and the prompt response to treatment, it seems reasonable to place the vaccination and the 'encephalitic state' in direct relationship. The incubation period of this condition is stated to be between nine and nineteen days-usually between the tenth and twelfth days, as in this case.

The treatment of post-vaccinial encephalitis is a matter in which there seems to be no very clear medical opinion. Brain (1940) reports ' encouraging results' by a number of authors following 'the injection of serum or citrated blood of a recently vaccinated person'; it is suggested that the serum may be given intrathecally, but that it may be equally effective intravenously in doses of 10 c.c. Recently, Burton and Weir (1941) successfully treated an infant of six months by giving 20 c.c. of paternal blood intramuscularly and later 20 c.c. of blood on four successive days from a nurse vaccinated two months previously: this treatment was supplemented by the administration of sulphapyridine parenterally and by mouth.

In the present case the most pressing indication for treatment was provided by the persistent restlessness, convulsions and respiratory distress which, in themselves, seemed to constitute a threat to life, and it is felt that the administration of pentothal sodium was the most valuable part of the treatment in that it provided rest where other measures had failed. Dehydration had also contributed to the moribund state and was relieved by intravenous fluid. It is difficult to assess the part played by the convalescent serum, which was given in much larger quantity than in previously reported cases, but it seems reasonable to suppose that it exerted a beneficial effect in the period following cessation of the convulsions. It is evident that large quantities of serum may be given without danger.

\section{Summary}

A case of post-vaccinial encephalitis in an infant of four months is reported. Recovery followed treatment with intravenous pentothal sodium and convalescent serum, and the value of the former in the treatment of infantile convulsions is emphasized. 
Thanks are due to Prof. C. W. Vining for his encouragement and permission to publish this case report.

\section{REFERENCES}

Brain, W. R. (1940). Recent Advances in Neurology, 4th edition, Lond., 251. Burton, A. H. G., and Weir, J. H. (1941). Lancet, 2, 561. 